\title{
Correct placement of the Essure device detected by transvaginal ultrasound at one month predicts correct placement at three months
}

\author{
Sarah Mitchell ${ }^{1}$, James May ${ }^{2}$, Alex Ades ${ }^{1} *$
}

\begin{abstract}
${ }^{1}$ Department of Obstetrics and Gynaecology, Royal Women's Hospital, 20 Flemington Rd, Parkville-3052, Australia
${ }^{2}$ Laparoscopic Surgery Fellow, Frances Perry House, 20 Flemington Rd, Parkville-3052, Australia
\end{abstract}

Received: 27 November 2013

Accepted: 15 December 2013

\section{*Correspondence:}

Dr. Alex Ades,

E-mail: alex@ades.com.au

(C) 2014 Mitchell S et al. This is an open-access article distributed under the terms of the Creative Commons Attribution Non-Commercial License, which permits unrestricted non-commercial use, distribution, and reproduction in any medium, provided the original work is properly cited.

\begin{abstract}
Background: Correct placement and tubal occlusion following Essure sterilisation is presently confirmed with imaging 3 months post procedure. Compliance with post procedure imaging is low and non-compliance has been implicated in unintended pregnancies following Essure insertion. Patient adherence to medical advice is known to decrease with time. Earlier imaging may improve compliance. The Aim was to determine if correct placement of the Essure micro-insert detected at 1 month by transvaginal ultrasound (TV US) is predictive of correct placement at 3 months.

Methods: Prospective cohort of 31 patients in a single centre who underwent the Essure procedure and had TV US at 1 month and 3 months post procedure.

Results: The Essure micro-insert was detected in the accurate position by TV US in all 31 women at both 1 month and 3 months post insertion.

Conclusions: Correct placement of the micro-insert detected at 1 month is predictive of correct placement at 3 months. TV US at any time from 1 month could be used as the confirmation test for correct placement of the Essure device. This may provide earlier reassurance to women and improve compliance with follow up potentially reducing numbers of unintended pregnancies. The importance of alternative contraception until 3 months post insertion should still be emphasised.
\end{abstract}

Keywords: Essure, Ultrasound, Contraception, Hysteroscopic sterilisation

\section{INTRODUCTION}

Hysteroscopic sterilisation is a minimally invasive, safe and reliable method of permanent birth control that has gained considerable popularity in recent times. The hysteroscopic route avoids the need for general anaesthesia, is well tolerated and can be performed in an office setting. ${ }^{1,2}$ Most women require minimal time off and can promptly return to work and normal daily activities. The Essure micro-insert (Essure Permanent Birth Control System, Conceptus, Inc., San Carlos, CA) was approved for use in hysteroscopic sterilisation by the US Food and Drug Administration in November 2002. The procedure involves inserting the Essure micro-coil into the proximal fallopian tube with a catheter delivery system under hysteroscopic guidance. The presence and material of the micro-coil induces a fibrotic reaction in the surrounding tissues which leads to tubal occlusion. ${ }^{3}$

Post procedure imaging tests are recommended after three months to confirm adequate device placement and/or tubal occlusion. In the United States, the FDA 
recommends that tubal occlusion be demonstrated and the test of choice is a hysterosalpingogram (HSG).

In other countries, including most of Western Europe and Australia, the test of choice is a transvaginal ultrasound. Several studies have demonstrated that the presence of the device in the interstitial portion of the Fallopian tubes amounts to tubal occlusion with no increase in failure rates and ultrasound is associated with much lower reported discomfort and pain than the HSG. ${ }^{4-8}$

The current recommendation for performing the confirmation test three months post-procedure is derived from a peri-hysterectomy study. Women undergoing elective hysterectomy agreed to have the Essure procedure prior to their surgery. The hysterectomy was performed at different time intervals after the Essure. All women had an HSG prior to the hysterectomy and all tubes were subsequently histologically examined. A large proportion of women had their tubes occluded after 6 to 8 weeks. After 12 weeks, tubal occlusion was noted in $100 \%$ of tubes. Furthermore, at least some degree of tissue reaction was observed in all specimens from as early as 1 week post procedure. ${ }^{3}$ Another study comparing contrast infusion sonography with HSG as a means of confirming tubal occlusion noted a high rate (82\%) of bilateral fallopian tube occlusion on HSG at just 4 weeks post Essure. ${ }^{9}$

Current practice in our hospital is to perform a transvaginal ultrasound 3 months after the Essure procedure.

Non-compliance with the post-procedure confirmation test is one factor implicated in unintended pregnancies following Essure insertion. ${ }^{10,11}$ Shavell et al. showed a $12.7 \%$ compliance with HSG 3-6 months post procedure despite preoperative and postoperative counselling and suggested that additional steps to promote compliance should be utilised. ${ }^{12}$ Long-term adherence to medical recommendations is a recognised problem in all aspects of medical practice. ${ }^{13,14}$ While factors influencing patient compliance are complex, reduced compliance over time is a well-established principle. Earlier placement detection has the potential to increase compliance, reduce the number of visits to doctors and reduce the number of unintended pregnancies thus improving method efficacy. In addition, Teoh et al. suggested that an early post insertion TV US may provide reassurance and reduce patient anxiety as well as provide an early diagnose in case of malposition. ${ }^{15}$

This study was conducted to verify whether an ultrasound performed at 1 month would have the same accuracy for predicting method efficacy as the one performed at 3 months.

\section{METHODS}

Between March 2008 and November 2011, women who underwent the Essure procedure were asked to participate in the study. Patients who agreed were asked to have an extra ultrasound 1 month post-procedure in addition to the routine 3 month follow up ultrasound. Thirty one women agreed to participate in the study.

In accordance with current recommendations, all women were advised to use alternative contraception until accurate placement was confirmed on TV US at 3 months.

All Essure procedures were performed at the Royal Women's Hospital in Melbourne by different specialist gynaecologists. All procedures were performed in theatre under general anaesthetic.

The surgical technique was the standard recommended by the manufacturer where a surgical hysteroscope with a 5 french working channel is used to pass the delivery catheter and insert the Essure device into the Fallopian tubes using defined visual markers. Adequate placement at the time of the procedure was defined as Essure device inside the tube with between 2 and 8 trailing coils inside the uterine cavity.

Correct placement on ultrasound was defined as the presence of the Essure micro-insert inside seen at the interstitial portion of the Fallopian tubes on transvaginal ultrasound.

\section{Demographics}

Patients age varied from 23 to 47 with a median of 33 years ( $\mathrm{SD}=4$ years). Parity varied from 2 to 6 children with an average of 2.3 children.

This review conforms to the standards established by the NHMRC for ethical quality review and the declaration of Helsinki. ${ }^{16}$

\section{RESULTS}

All patients had successful placement at the time of surgery. No surgical complications were reported.

All 31 women had correct positioning of the micro-insert identified at 1 month and again at 3 months post insertion.

All patients were followed up for a minimum of 24 months, maximum 48 months. No pregnancies were reported. 


\section{DISCUSSION}

Transvaginal ultrasound is a widely accepted test used as confirmation for placement of Essure micro-inserts. The ultrasound is not a test of tubal occlusion but demonstrates the presence of the micro-insert and accurately determines its position inside the Fallopian tube. It has been demonstrated that micro-insert presence inside the interstitial portion of the Fallopian tube can be used as a proxy for tubal occlusion. ${ }^{4-8}$

The ability to perform the test sooner than the currently recommended three months post procedure has the potential to increase patient compliance and reassure patients earlier of correct micro-insert placement.

In this study, all women who had correct placement identified at 1 month post procedure also had correct placement confirmed at 3 months. Therefore, we propose that correct placement on TV US at any time after 1 month can be used as the confirmation test for correct placement of the Essure devices.

The authors do not suggest that a sufficient level of tubal occlusion has taken place for sterilisation to be effective at 1 month in all patients. Accordingly, the current advice for alternative contraception for 3 months post procedure should still be emphasized.

A simple follow up schedule for patients who underwent Essure hysteroscopic sterilisation can be achieved with one single outpatient appointment between 4 to 6 weeks where an office transvaginal ultrasound is performed. The woman can be told then that her procedure was successful and she will be able to rely solely on Essure for contraception at 3 months. As mentioned, continuation of the currently used alternative contraceptive method until the end of the 3 months should be stressed at this review appointment.

The number of patients in the study is relatively small; however our results are very encouraging in suggesting that the transvaginal ultrasound for confirmation of proper placement of Essure micro-inserts can be done at any time from 1 month after the procedure. This offers the potential to improve compliance with the postprocedure confirmation test, a factor implicated in unintended pregnancies following Essure insertion, and reduce anxiety by reassuring women earlier that the procedure was successful. Further studies with larger number of women and with ultrasounds performed at different times after the procedure could confirm these findings.

Funding: No funding sources Conflict of interest: None declared

Ethical approval: Not required

\section{REFERENCES}

1. Ariona Je, Miño M, Cordón J, Povendano B, Pelegrin B, Castelo-Branco C. Satisfaction and tolerance with office hysteroscopic tubal sterilisation. Fertil Steril. 2008;90:1182-6.

2. Wortman M, Daggett A, Ball C. Operative hysteroscopy in an office-based surgical setting: review of patient safety and satisfaction in 414 cases. J Minim Invasive Gynecol. 2013;20:56-63.

3. Valle RF, Carignan CS, Wright TC. Tissue response to the STOP microcoil transcervical permanent contraceptive device: results from a prehysterectomy study. Fertil Steril. 2001;76:974-80.

4. Theil J, Suchet I, Tyson N, Price P. Outcomes in the ultrasound follow-up of the Essure micro-insert: complications and proper placement. J Obstet Gynaecol Can. 2011;33:134-8.

5. Verseema S, Vleugels M, Koks C, Thurkow A, van der Vaart H, Brölmann H. Confirmation of Essure placement using transvaginal ultrasound. J Minim Invasive Gynecol. 2011;18:164-8.

6. Weston G, Bowditch J. Office ultrasound should be the first-line investigation for confirmation of correct Essure placement. Aust N Z J Obstet Gynaecol. 2005;45:312-5.

7. Kerin JF, Levy BS. Ultrasound: an effective method for localisation of echogenic Essure sterilisation micro-insert: correlation with radiologic evaluations. J Minim Invasive Gynecol. 2005;12:50-4.

8. Chapa, HO, Bakker K, Sandate J, Silver L, Antonetti AG. Essure sterilisation with sonographic confirmation test: protocol description and clinical outcomes using ultrasound as an Essure confirmation test. J Gynecol Surg. 2011;27:57-62.

9. Connor V. Contrast infusion sonography in the postEssure setting. J Minim Invasive Gynecol. 2008; 15:56-61

10. Jost S, Huchon C, Legendre G, Letohic A, Fernandez H, Panel P. Essure(®) permanent birth control effectiveness: a seven-year survey. Eur J Obstet Gynecol Reprod Biol. 2013Jun;168:134-7.

11. Veersema S, Vleugels MP, Moolenaar LM, Janssen CA, Brölmann HA Unintended pregnancies after Essure sterilization in the Netherlands. Fertil Steril. 2010;93:35-8.

12. Shavell VI, Abdallah ME, Diamond MP, Kmak DC, Berman JM. Post-Essure hysterosalpingography compliance in a clinic population. $\mathrm{J}$ Minim Invasive Gynecol. 2008;15:431-4.

13. Soutter WP, Moss B, Perryman K, Kyrgiou M, Papakonstantinou K, Ghaem-Maghami S. Long-term compliance with follow-up after treatment for cervical intra-epithelial neoplasia. Acta Obstet Gynecol Scand. 2012Sep;91:1103-8.

14. Benner JS, Glynn RJ, Mogun H, Neumann PJ, Weinstein MC, Avorn J. Long-term persistence in use of statin therapy in elderly patients. JAMA. 2002Jul24-31;288:455-61. 
15. Teoh M, Meagher S, Kovacs G. Ultrasound detection of the Essure permanent birth control device: a case series. Aust N Z Obstet Gynaecol. 2003;43:378-80.

16. National Health and Medical Research Council. National Statement of Ethical Conduct in Human Research. The National Health and Medical Research Council, the Australian Research Council and the Australian Vice-Chancellors' Committee. Commonwealth of Australia, Canberra. 2007 Available

at: http://www.ambulance.vic.gov.au/media/docs/e72_n ational_statement_130624-028bd263-b454-4088a6d0-64eff4ce07d2-0.pdf. Accessed May 2013.

DOI: $10.5455 / 2320-1770 . i j r \operatorname{cog} 20140315$

Cite this article as: Mitchell S, May J, Ades A. Correct placement of the Essure device detected by transvaginal ultrasound at one month predicts correct placement at three months. Int J Reprod Contracept Obstet Gynecol 2014;3:75-8. 